\title{
A Psychometric Comparison of Two Carer Quality of Life Questionnaires in Huntington's Disease: Implications for Neurodegenerative Disorders - A response to: Hagell and Smith (2013) Journal of Huntington's Disease 2(3) 315-322
}

\author{
A. Aubeeluck, H. Buchanan and E. Stupple
}

Dear Editor,

Hagell and Smith (H\&S, [6]) present an analysis of the Huntington's Disease Quality of Life Battery for Carers (HDQOL-C) to test and compare it with the Alzheimer's Carers Quality of Life Inventory. They claim the HDQOL-C "fail[s] to meet basic psychometric properties" (p. 315) and argue for "the feasibility of a common neurodegenerative carer QoL questionnaire" (p. 319). We feel their dismissal of the need for a scale that examines the unique disease-specific issues that $\mathrm{HD}$ carers face, is unjustified. We question their characterisation of the HDQoL-C, methodology and analyses, and argue that their dataset fails to meet basic psychometric criteria.

A small sample $(n=$ approximately 45 when accounting for missing data), from one clinic, in one geographical location is unlikely to be generalisable to the wider HD carer population. It is not surprising that the HDQoL-C subscales do not fare well when tested on such a homogenous data set, particularly as some items evaluate standards of support available in the carer's location. Moreover, replication studies should match or exceed the sample size of previous work.
Our previous data ( $1: n=87 ; 2: n=301)$, provide a far superior sample of carers from across the EU. Psychometric evaluation usually includes factor analysis and standard tests of validity. $\mathrm{H} \& \mathrm{~S}$ present under-powered and predominantly descriptive analyses from a data set that does not meet standard psychometric criteria. Therefore, H\&S's claim that the HDQoL-C is not psychometrically sound is difficult to justify.

H\&S challenge the HDQoL's "suitability among HD carers" (p. 319), and "user friendliness" (p. 319) based on completion rates. In comparison studies, counterbalancing of scale completion is essential, but was not reported by H\&S. We have found better completion rates in our studies and would need convincing that there was more to these differences than random fluctuation in their sample. Indeed, carers in our studies have reported that completing the HDQOL-C is a positive and therapeutic experience that offers opportunities for self-reflection.

The development of the HDQoL-C is poorly described. Claims that "The HDQOL-C fails to provide an explicit definition for its target variables" (p. 320) and "content validity was not informed by carers, but 
by QoL and HD experts" (p. 320) are inaccurate. The HDQoL- $\mathrm{C}$ is grounded in psychometric theory and was developed based on the WHO definition of Quality of life. Carers were considered experts of their own experience and were involved throughout the development of the HDQOL-C $[1,3,4]$. This misunderstanding by $H \& S$ appears to contribute to their dismissal of the need for a HD-specific measure.

The HDQoL-C gives carers a way of quantifying their experience of living with HD and has been beneficial within clinical practice, on an individual level and in HD carer research (e.g. 5). We argue that a generic scale could not identify disease-specific issues which in turn, could significantly impact on tailored support for this unique group. Moreover, H\&S's preferred scale is behind a pay wall with no peer reviewed validation paper available in the public domain. The HDQoL$\mathrm{C}$ and its psychometric properties are freely available for families, clinicians and researchers to access and use for the benefit of improving the quality of life of families living with HD (http://www.nottingham. ac.uk/nmpresearch/hdqol-c/documents.aspx).

We welcome robust and objective psychometric evaluation of our scale and have an increasing dataset that researchers who have requested permission to use the scale, have kindly shared with us. We have extended this invitation to $\mathrm{H} \& \mathrm{~S}$, and would be happy to include their data within a more robust analysis of the HDQOL$\mathrm{C}$ in a larger cohort of participants.

\section{REFERENCES}

[1] Aubeeluck A, Buchanan H. The Huntington's Disease quality of life battery for carers: Reliability and validity. Clinical Genetics. 2007;71(5):434-45.

[2] Aubeeluck A, Dorey J, Ferdinando S, Clay E, Stupple EJN, Annunziata N, Buchanan H, Tiziana M, Toumi M. Further evidence of reliability and Validity of the Huntington's disease quality of life battery for carers: Italian and French translations. Quality of Life Research. 2012;22(5):1093-8.

[3] Aubeeluck A. Spousal caregiving in Huntington's disease: An issue for health psychology. Health Psychology Update. 2003;12(4):20-7.

[4] Aubeeluck A. A measure to assess the impact of Huntington's disease on the quality of life of spousal carers. British Journal of Neuroscience Nursing. 2006;2(2):86-93.

[5] Cox M. Quality of life among carers of individuals with Huntington's disease. British Journal of Neuroscience Nursing. 2012;5(5):288-95.

[6] Hagell P, Smith S. A psychometric comparison of two carer quality of life questionnaires in Huntington's disease: Implications for neurodegenerative disorders. Journal of Huntington's Disease. 2013;2(3):315-22. 